\title{
Two types of mental fatigue affect spontaneous oscillatory brain activities in different ways
}

\author{
Yoshihito Shigihara ${ }^{1}$, Masaaki Tanaka ${ }^{1 *}$, Akira Ishii $^{1}$, Etsuko Kanai ${ }^{2}$, Masami Funakura ${ }^{2}$ and Yasuyoshi Watanabe $^{1,3}$
}

\begin{abstract}
Background: Fatigue has a multi-factorial nature. We examined the effects of two types of mental fatigue on spontaneous oscillatory brain activity using magnetoencephalography (MEG).

Methods: Participants were randomly assigned to two groups in a single-blinded, crossover fashion to perform two types of mental fatigue-inducing experiments. Each experiment consisted of a 30-min fatigue-inducing 0- or 2-back test session and two evaluation sessions performed just before and after the fatigue-inducing mental task session.

Results: After the 0-back test, decreased alpha power was indicated in the right angular gyrus and increased levels in the left middle and superior temporal gyrus, left postcentral gyrus, right superior frontal gyrus, left inferior frontal gyrus, and right medial frontal gyrus. After the 2-back test, decreased alpha power was indicated in the right middle and superior frontal gyrus and increased levels in the left inferior parietal and superior parietal lobules, right parahippocampal gyrus, right uncus, left postcentral gyrus, left middle frontal gyrus, and right inferior frontal gyrus. For beta power, increased power following the 0-back test was indicated in the left middle temporal gyrus, left superior frontal gyrus, left cingulate gyrus, and left precentral gyrus. After the 2-back test, decreased power was suggested in the left superior frontal gyrus and increased levels in the left middle temporal gyrus and left inferior parietal lobule. Some of these brain regions might be associated with task performance during the fatigue-inducing trials.
\end{abstract}

Conclusions: Two types of mental fatigue may produce different alterations of the spontaneous oscillatory MEG activities. Our findings would provide new perspectives on the neural mechanisms underlying mental fatigue.

Keywords: Magnetoencephalography (MEG), Mental fatigue, n-back test visual analogue scale (VAS)

\section{Background}

Fatigue refers to the feeling that people may experience after or during prolonged periods of activity [1] and is a common problem in modern society [2]. Since fatigue decreases efficiency in the performance of daily activities, clarifying the mechanisms underlying fatigue and developing efficient methods for overcoming it would be beneficial. The neural mechanisms of mental fatigue are to some extent clarified: From the results of the studies using event-related potential, evaluation of predicted rewards and potential risks of actions were considered to be central to the phenomenon of mental fatigue and the evaluation system was considered to consist of a neural circuit that interconnects basal ganglia, amygdala,

\footnotetext{
* Correspondence: masa-t@msic.med.osaka-cu.ac.jp

'Department of Physiology, Osaka City University Graduate School of Medicine, 1-4-3 Asahimachi, Abeno-ku, Osaka City, Osaka 545-8585, Japan Full list of author information is available at the end of the article
}

insular cortex, orbitofrontal cortex, prefrontal cortex, and anterior cingulate cortex [1]; in addition, the brain region associated with the sense of fatigue was identified as the orbitofrontal cortex [3].

Although definition of fatigue is not uniform, there seems to exist a common concept that fatigue is related to the impairment of performance rather than fatigue sensation [4-6]. Fatigue sensation is not fatigue itself, since fatigue sensation is involved in the biological alarm to order rest under the condition of fatigue; while fatigue itself is involved in the neural damage manifested as impaired performance [2]. Today, little is known about the neural mechanisms of mental fatigue related to the performance.

Mental fatigue is observed as a reduced efficiency for mental tasks [5]. Recently, new methods of induction and evaluation of mental fatigue have been proposed

\section{Biomed Central}


[7-9]. As a fatigue-inducing mental task session, participants performed 0 - or 2-back test trials for $30 \mathrm{~min}$. The 0-back test was used to represent a lower mental-load task, which could be performed without the use of working memory, while the 2-back test was used to represent a higher mental-load task, which required working memory [10]. The advantage of using these tasks is in their ability to cause two types of mental fatigue: After both 0-back and 2-back test sessions, impairment of task performance assessed by the percentage of correct task trials was significant, while longer reaction times and a higher subjective level of sleepiness could be identified only after the 0 -back test trials. Since mental fatigue is multi-faceted [11], using two types of mental tasks might reveal different facets.

Although a variety of psychophysiological parameters have been used in previous research dealing with fatigue, electroencephalography (EEG) has been proposed as a promising indicator of mental fatigue [12]. The electrical activity of the brain is classified according to rhythms defined according to frequency bands, including beta, alpha, theta, and delta. Each frequency band is associated with specific information processing in the central nervous system [13]. Recently, we found that two types of mental fatigue affected spontaneous EEG alpha and beta power densities in different way [14]: After enrollment, 18 participants were randomly assigned to two groups in a single-blinded, crossover fashion to perform two types of mental fatigue-inducing experiments. Each experiment consisted of four 30-min fatigue-inducing 0 or 2-back test sessions and two evaluation sessions performed just before and after the fatigue-inducing sessions. Eleven electrodes were attached to the head skin, from positions $\mathrm{F} 3, \mathrm{Fz}, \mathrm{F} 4, \mathrm{C} 3, \mathrm{Cz}, \mathrm{C} 4, \mathrm{P} 3, \mathrm{Pz}, \mathrm{P} 4, \mathrm{O} 1$, and $\mathrm{O} 2$. In the 2-back test, the beta power densities on the $\mathrm{P} 3, \mathrm{Pz}$, and $\mathrm{O} 1$ electrodes and the alpha power densities on the P3, Pz, O1, and O2 electrodes were decreased, and the theta power densities on the $\mathrm{Fz}$ and $\mathrm{Cz}$ electrodes were increased after the fatigue-inducing mental task sessions. In the 0-back test, the alpha power densities on the P3 and Pz electrodes were decreased after the fatigue-inducing sessions. However, because of limited spatial resolution, it was impossible to identify the brain regions associated with the alteration of the EEG power densities. In addition, the roles of the EEG power changes caused by mental fatigue were unclear. Compared to EEG, magnetoencephalography (MEG) has the following advantages: (1) the magnetic field is hardly affected by intervening tissues; (2) measurements of the magnetic field do not require a reference; and (3) measurements from many sensors can be performed [15]. MEG shares with EEG the advantages of measuring brain activity by using time-frequency analyses and having a high temporal resolution. Therefore, alterations of spontaneous MEG alpha and beta power densities induced by mental fatigue may provide valuable clues to identify the neural mechanisms of fatigue. The aim of our study was thus to clarify how the neural underpinnings of mental fatigue relate to task performance using MEG focusing on the resting-state time-frequency analyses of the alpha and beta frequency bands.

\section{Methods}

\section{Participants}

Ten male healthy volunteers $[30.8 \pm 9.4$ years of age (mean $\pm \mathrm{SD})$ ] were enrolled in this study. They had normal or corrected-to-normal visual acuity, no history of medical illness, and were right-handed according to the Edinburgh handedness inventory [16]. The study protocol was approved by the Ethics Committee of Osaka City University, and all the participants gave written informed consent for participation in this study.

\section{Experimental design}

This study was composed of two experiments in order to compare two types of mental fatigue. After enrollment, the participants were randomly assigned to two groups in a single-blinded, crossover fashion to perform two types of fatigue-inducing mental tasks (the same participants performed both 0 - and 2-back tasks and that order of task was counterbalanced, i.e., five participants began with the 0-back test and the others with the 2-back test). An experiment was composed of three sessions: one fatigue-inducing mental task session and two evaluation sessions (Figure 1). During the fatigueinducing mental task session, they performed 0-back or 2-back test trials for $30 \mathrm{~min}$ [10]. Just before and after the fatigue-inducing mental task session, MEG recordings were performed in the evaluation sessions with the subject's eyes closed for $1 \mathrm{~min}$ in relaxed wakefulness, and subjective scaling using visual analogue scales (VAS's) was performed before the first MEG recording and after the second recording. This study was conducted in a magnetically shielded room at Osaka City University Hospital. For 1 day before each visit, they refrained from intense mental and physical activities and caffeinated beverages, consumed a normal diet, and maintained normal sleeping hours. The healthy volunteers had not taken any form of medication during the last week. The time interval between the two experiments was $24 \mathrm{hr}$.

\section{Fatigue-inducing mental tasks}

Participants performed a 0-back or 2-back test for $30 \mathrm{~min}$ on a bed of a MEG scanner lying in supine position during the fatigue-inducing mental task session. They watched a video projection screen located $30 \mathrm{~cm}$ straight ahead of their eyes. Any of four types of letters 


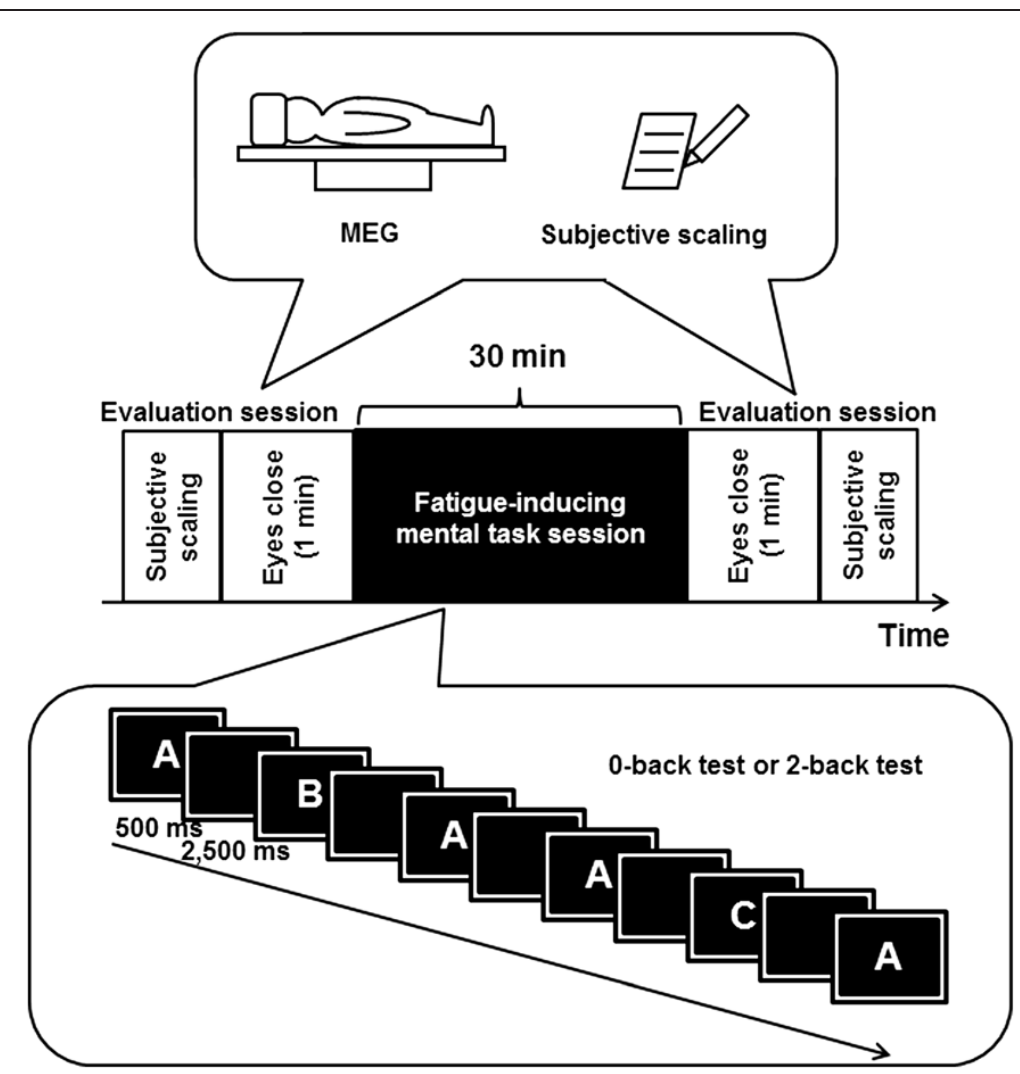

Figure 1 Experimental design. Each experiment consisted of one fatigue-inducing mental task session and two evaluation sessions performed just before and after the fatigue-inducing mental task session. During the fatigue-inducing mental task session, participants performed 0-back or 2-back test trials for $30 \mathrm{~min}$. They were randomly assigned to two groups in a single-blinded, crossover fashion to perform the two types of the fatigue-inducing trials on separate days (the same participants performed both 0- and 2-back tasks and that order of task was counterbalanced, i.e., five participants began with the 0-back test and the others with the 2-back test). During the evaluation sessions, magnetoencephalography (MEG) recordings were performed with the participant's eyes closed and subjective scaling.

were continually presented on the video screen every $3 \mathrm{sec}$. The letter was presented on the screen for $500 \mathrm{~ms}$ and not presented for $2500 \mathrm{~ms}$ thereafter. During the 0 -back trials, they were asked to press the right button with their right middle finger if the target letter (shown beside the video screen) was presented at the center of the video screen. If any other letters appeared, they were to press the left button with their right index finger. During the 2-back trials, they had to judge whether the target letter presented at the center of the video screen was the same as the one that had appeared 2 presentations before. If it was, they were to press the right button with their right middle finger, while if it was not they were to press the left button with their right index finger. They were instructed to perform the task trials as quickly and as correctly as possible. The result of each 0-back or 2-back trial - correct response or error - was continually presented on the display of the video screen. Performance on these tests was evaluated through the percentage correct and mean reaction time. To evaluate the time course of task performance during the fatigue- inducing mental task session, the performance measures were calculated every $5 \mathrm{~min}$.

\section{Subjective scaling}

Subjective scaling was performed to evaluate participants' subjective levels of fatigue and sleepiness. The participants were verbally asked to rate their subjective levels of fatigue (in Japanese, tsukareteiru) and sleepiness (in Japanese, nemui) on a VAS from 0 (minimum) to 100 (maximum) using a paper-and pencil questionnaire [17].

\section{MEG recordings}

MEG recordings were performed using a 160-channel whole-head type MEG system (MEG vision; Yokogawa Electric Corporation, Tokyo, Japan) with a magnetic field resolution of $4 \mathrm{fT} / \mathrm{Hz}^{1 / 2}$ in the white-noise region. The sensor and reference coils were gradiometers $15.5 \mathrm{~mm}$ in diameter and $50 \mathrm{~mm}$ in baseline, and each pair of sensor coils was separated at a distance of $23 \mathrm{~mm}$. The sampling rate was $1,000 \mathrm{~Hz}$ with a $1 \mathrm{~Hz}$ high-pass filter. 


\section{MEG data analyses}

MEG signal data were analyzed offline after analogue-todigital conversion. Epochs of the raw MEG data including artifacts such as running trains of subway were excluded from the analysis by careful visual detection of the artifacts before averaging. After averaging, the power was determined in two frequency bands, alpha $(8-13 \mathrm{~Hz})$ and beta $(13-25 \mathrm{~Hz})$ by a fast Fourier transformation using Frequency Trend (Yokogawa Electric Corporation) for each participant and the contrast images of (after the n-back test trials)/(before the n-back test trials) was constructed for each frequency band.

Anatomical magnetic resonance imaging (MRI) was performed using a Philips Achieva 3.0TX (Royal Philips Electronics, Eindhoven, Netherland) to permit registration of magnetic source locations with their respective anatomical locations. Before MRI scanning, five adhesive markers (Medtronic Surgical Navigation Technologies Inc., Broomfield, $\mathrm{CO}$ ) were attached to the skin of the participant's head (the first and second ones were located at $10 \mathrm{~mm}$ in front of the left tragus and right tragus, the third at $35 \mathrm{~mm}$ above the nasion, and the fourth and fifth at $40 \mathrm{~mm}$ to the right and left of the third one). The MEG data were superimposed on MR images using information obtained from these markers and the MEG localization coils. Localization and intensity of the timefrequency power of the cortical activity were estimated using the software Brain Rhythmic Analysis for MEG (BRAM; Yokogawa Electric Corporation) [18], which used narrow-band adaptive spatial filtering technique. These data were then analyzed using Matlab (Mathworks, Sherbon, MA), implemented in statistical parametric mapping (SPM8, Wellcome Department of Cognitive Neurology, London, UK). The MEG anatomical/spatial parameters used to warp the volumetric data were transformed into the Montreal Neurological Institute (MNI) template of T1-weighed images [19] and applied to the MEG data. The anatomically normalized MEG data were filtered with a Gaussian kernel of $15 \mathrm{~mm}$ (full-width at half-maximum) in the $\mathrm{x}, \mathrm{y}$, and $\mathrm{z}$ axes. The oscillatory power for each frequency band after the n-back test trials relative to that before the nback test trials was measured on a region-of-interest basis. The resulting set of voxel values for each comparison constituted a SPM of the $t$ statistics $(\operatorname{SPM}\{t\})$. The $\operatorname{SPM}\{t\}$ was transformed to the unit of normal distribution $(\operatorname{SPM}\{Z\})$. The threshold for the $\operatorname{SPM}\{Z\}$ of individual analyses was set at $P<0.001$ (uncorrected for multiple comparisons). The weighted sum of the parameters estimated in the individual analyses consisted of "contrast" images, which were used for the group analyses [20]. So that inferences could be made at a population level, individual data were summarized and incorporated into a random-effect model [20]. SPM $\{t\}$ and $\operatorname{SPM}\{Z\}$ for the contrast images were created as described above. Significant signal changes for each contrast were assessed by means of $t$ statistics on a voxelby-voxel basis [20]. The threshold for the $\operatorname{SPM}\{Z\}$ of group analyses was set at $P<0.001$ (uncorrected for multiple comparisons). Anatomical localizations of significant voxels within clusters were done using the Talairach Demon software [21] with the nearest gray matter option enabled. We captured the fiducial coils and tracked the head position before and after the MEG recording. The criterion for head movement exclusion was the movement more than one voxel size $(5 \times 5 \times$ $5 \mathrm{~mm})$.

\section{Statistical analyses}

Data are presented as mean \pm SD unless otherwise stated. Two-way analyses of variance (ANOVA's) for repeated measures were performed to evaluate the significance of difference among VAS values. Paired $t$-test was used to evaluate the significance of differences between two conditions. Simple regression analyses were conducted to evaluate the relationships between task performances and MEG variables. All $\mathrm{P}$ values were two-tailed, and values less than 0.05 were considered to be statistically significant. Statistical analyses were performed using the SPSS 20.0 software package (SPSS, Chicago, IL).

\section{Results}

The task performance measures during 0-back and 2back test trials are shown in Figure 2. Although the percentage correct of the 2-back test was not different from that of the 0-back test, the reaction time of the 2-back test was longer than that of the 0-back test.

The subjective level of fatigue was significantly affected by time course (pre- and post-task measurement) $[\mathrm{F}(1,9)=18.05, \mathrm{P}<0.001]$, but not by task or by a task $\times$ time course interaction effect $[\mathrm{F}(1,9)=0.09, \mathrm{P}=0.766]$. No significant main effects were found for subjective level of sleepiness.

Alpha power changes after the fatigue-inducing mental task sessions are shown in Table 1 and Figure 3. After the 0-back test, decreased power was shown in the right angular gyrus [Brodmann's (BA) area 39] and increased levels were shown in the left middle temporal gyrus (BA 21 ), left superior temporal gyrus (BA 41), left postcentral gyrus (BAs 2 and 3), right superior frontal gyrus (BA 6), left inferior frontal gyrus (BA 47), and right medial frontal gyrus (BA 10). After the 2-back test, decreased power was shown in the right middle frontal gyrus (BAs 8 and 46) and right superior frontal gyrus (BAs 6 and 9) and increased levels were shown in the left inferior parietal lobule (BA 39), left superior parietal lobule (BA 7), right parahippocampal gyrus (BA 36), right uncus (BA 


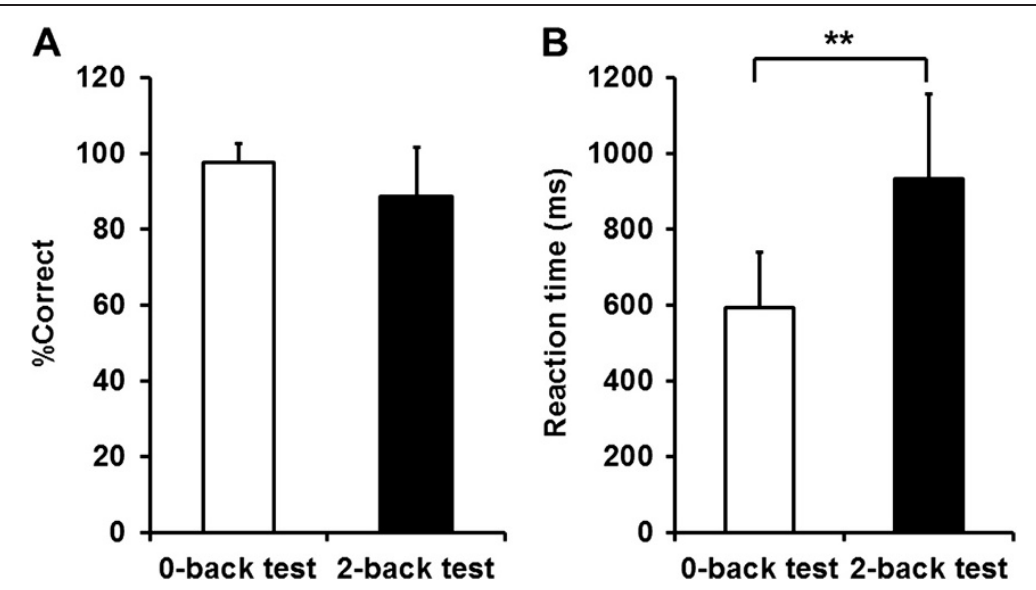

Figure 2 Task performance during 0-back and 2-back test trials. Rate of correct trials [\% correct] (A) and reaction time (B) were assessed. Values are presented as the mean and SD. ${ }^{* *} \mathrm{P}<0.01$, significant difference (paired $t$-test).

Table 1 Alpha power changes after fatigue-inducing task trials

\begin{tabular}{|c|c|c|c|c|c|c|}
\hline \multirow[t]{2}{*}{ Location } & \multirow[t]{2}{*}{ Side } & \multirow{2}{*}{$\begin{array}{c}\text { Brodmann's } \\
\text { area }\end{array}$} & \multicolumn{3}{|c|}{ Coordinates (mm) } & \multirow{2}{*}{$\begin{array}{c}\mathrm{Z} \\
\text {-value }\end{array}$} \\
\hline & & & $x$ & $y$ & $z$ & \\
\hline \multicolumn{7}{|l|}{ 0-back test } \\
\hline \multicolumn{7}{|l|}{ Decrease } \\
\hline Angular gyrus & $\mathrm{R}$ & 39 & 42 & -52 & 30 & 3.23 \\
\hline \multicolumn{7}{|l|}{ Increase } \\
\hline Middle temporal gyrus & L & 21 & -68 & -32 & -5 & 3.61 \\
\hline Middle temporal gyrus & $L$ & 21 & -68 & -52 & 0 & 3.43 \\
\hline Superior temporal gyrus & L & 41 & -48 & -22 & 10 & 3.39 \\
\hline Postcentral gyrus & L & 2 & -58 & -17 & 45 & 3.45 \\
\hline Postcentral gyrus & L & 3 & -53 & -7 & 40 & 3.34 \\
\hline Superior frontal gyrus & $\mathrm{R}$ & 6 & 22 & 38 & 55 & 3.18 \\
\hline Inferior frontal gyrus & L & 47 & -43 & 33 & -15 & 3.15 \\
\hline Medial frontal gyrus & $\mathrm{R}$ & 10 & 2 & 68 & 10 & 3.13 \\
\hline
\end{tabular}

\section{2-back test}

\section{Decrease}

Middle frontal gyrus

Middle frontal gyrus

Superior frontal gyrus

Superior frontal gyrus

\section{Increase}

Inferior parietal lobule

Superior parietal lobule

Parahippocampal gyrus

Uncus

Postcentral gyrus

Middle frontal gyrus

Middle frontal gyrus

Inferior frontal gyrus

$R$
$R$
$R$
$R$

52

42

17

27

$-53$

$-33$

27

32

$-53$

-53
-28

-28
-48

27 z alue

(a)




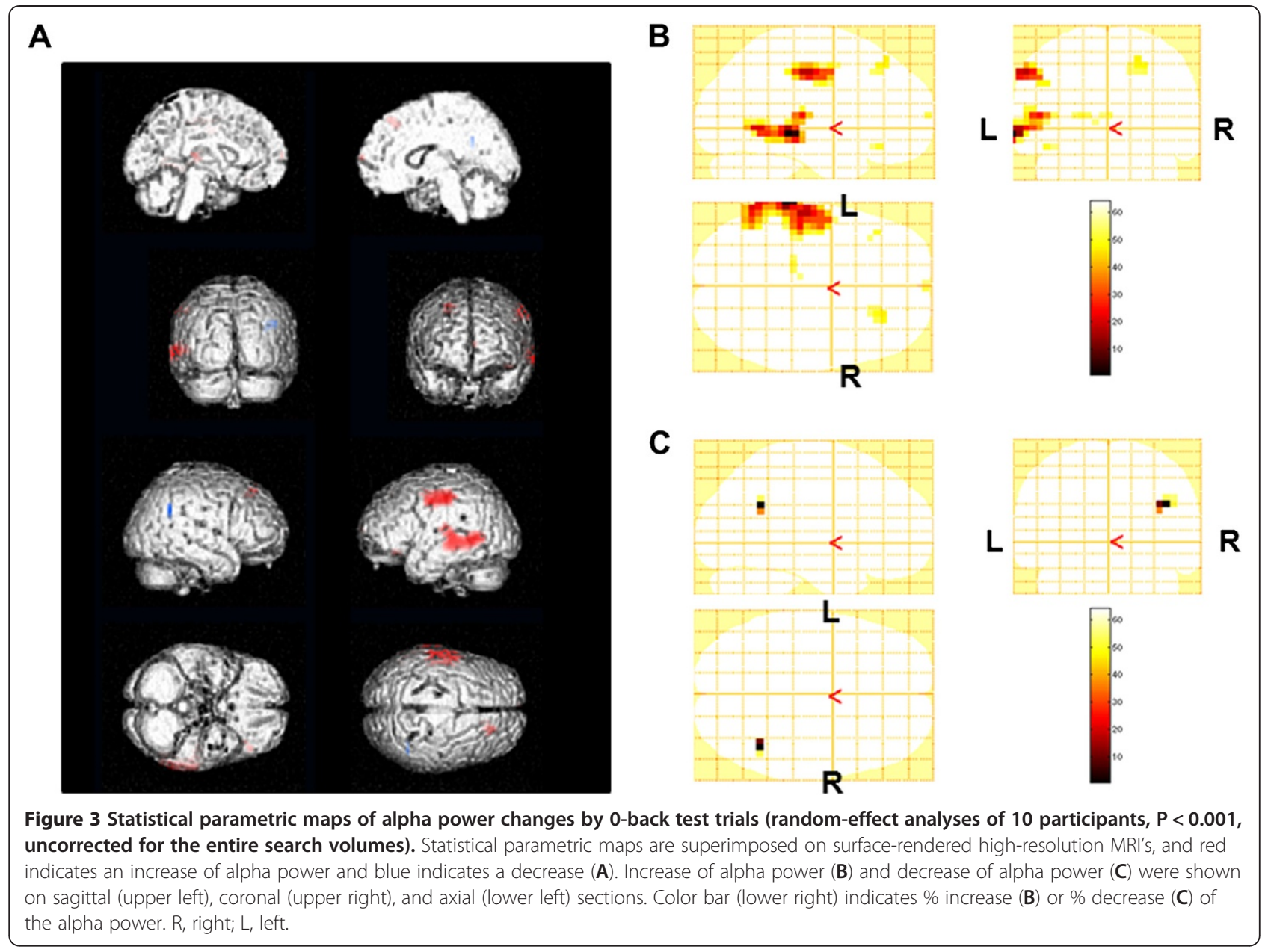

Table 2 Beta power changes after fatigue-inducing task trials

\begin{tabular}{|c|c|c|c|c|c|c|}
\hline \multirow[t]{2}{*}{ Location } & \multirow[t]{2}{*}{ Side } & \multirow{2}{*}{$\begin{array}{l}\text { Brodmann's } \\
\text { area }\end{array}$} & \multicolumn{3}{|c|}{ Coordinates (mm) } & \multirow{2}{*}{$\begin{array}{c}\mathrm{Z} \\
- \text { value }\end{array}$} \\
\hline & & & $x$ & $y$ & $z$ & \\
\hline \multicolumn{7}{|l|}{ 0-back test } \\
\hline \multicolumn{7}{|l|}{ Decrease } \\
\hline \multicolumn{7}{|l|}{ Increase } \\
\hline Middle temporal gyrus & L & 21 & -63 & -37 & -5 & 3.89 \\
\hline Superior frontal gyrus & L & 8 & -13 & 43 & 50 & 3.45 \\
\hline Cingulate gyrus & L & 31 & -23 & -42 & 20 & 3.30 \\
\hline Precentral gyrus & L & 4,6 & -63 & 8 & 30 & 3.09 \\
\hline \multicolumn{7}{|l|}{ 2-back test } \\
\hline \multicolumn{7}{|l|}{ Decrease } \\
\hline Superior frontal gyrus & L & 6 & -18 & 38 & 55 & 3.21 \\
\hline Superior frontal gyrus & L & 9 & -28 & 58 & 25 & 3.18 \\
\hline \multicolumn{7}{|l|}{ Increase } \\
\hline Middle temporal gyrus & L & 39 & -63 & -62 & 5 & 3.24 \\
\hline Inferior parietal lobule & $L$ & 40 & -48 & -52 & 35 & 3.18 \\
\hline
\end{tabular}

$\mathrm{x}, \mathrm{y}, \mathrm{z}$ : Stereotaxic coordinates.

Random-effect analyses of 10 participants $(P<0.001$, uncorrected for the entire search volumes). 
20), left postcentral gyrus (BA 3), left middle frontal gyrus (BA's 6 and 10), and right inferior frontal gyrus (BA 40).

Beta power changes after the fatigue-inducing mental task sessions are shown in Table 2 and Figure 4. After the 0-back test, increased levels were shown in the left middle temporal gyrus (BA 21), left superior frontal gyrus (BA 8), left cingulate gyrus (BA 31), and left precentral gyrus (BA 6). After the 2-back test, decreased power was shown in the left superior frontal gyrus (BA's 6 and 9) and increased levels were shown in the left middle temporal gyrus (BA 39) and left inferior parietal lobule (BA 40).

The alpha power changes in the right middle frontal gyrus (BA 46) and right superior frontal gyrus (BA 9) for the 2-back test session were lower than those for the 0back test session. The beta power change in the left precentral gyrus for the 2-back test session was lower than that for the 0-back test session. In order to find the brain regions related specifically to task performance, we performed correlation analyses. The alpha power changes in the right middle frontal gyrus (BA 46) and right superior frontal gyrus (BA 9) were negatively associated with the change of percentage correct (Figures 5A and $6 \mathrm{~A}, \mathrm{R}=0.655, \mathrm{P}=0.040$ for the right middle frontal gyrus; Figures $5 \mathrm{~B}$ and $6 \mathrm{~B}, \mathrm{R}=0.774, \mathrm{P}=0.009$ for the right superior frontal gyrus) for the 2-back test session. The beta power change in the left precentral gyrus (BA $4,6)$ was negatively associated with the change in percentage correct (Figures 7 and $8, \mathrm{R}=-0.755, \mathrm{P}=0.019$ ) and positively associated with the change of reaction time (Figures 7 and $8, \mathrm{R}=0.789, \mathrm{P}=0.011$ ) for the 0-back test session. We also performed the analyses within the delta and theta frequency bands. However, we could not identify the MEG results related to the task performance.

\section{Discussion}

Oscillatory brain rhythms are considered to originate from synchronous synaptic activities of a large number of neurons [22]. Synchronization of neural networks may reflect integration of information processing, and such synchronization processes can be evaluated using MEG time-frequency analyses [23]. Aberrant restingstate oscillatory activities have been observed in various pathological conditions $[15,24]$. We found that, after the 0-back test, decreased alpha power was shown in the angular gyrus and increased levels were shown in the

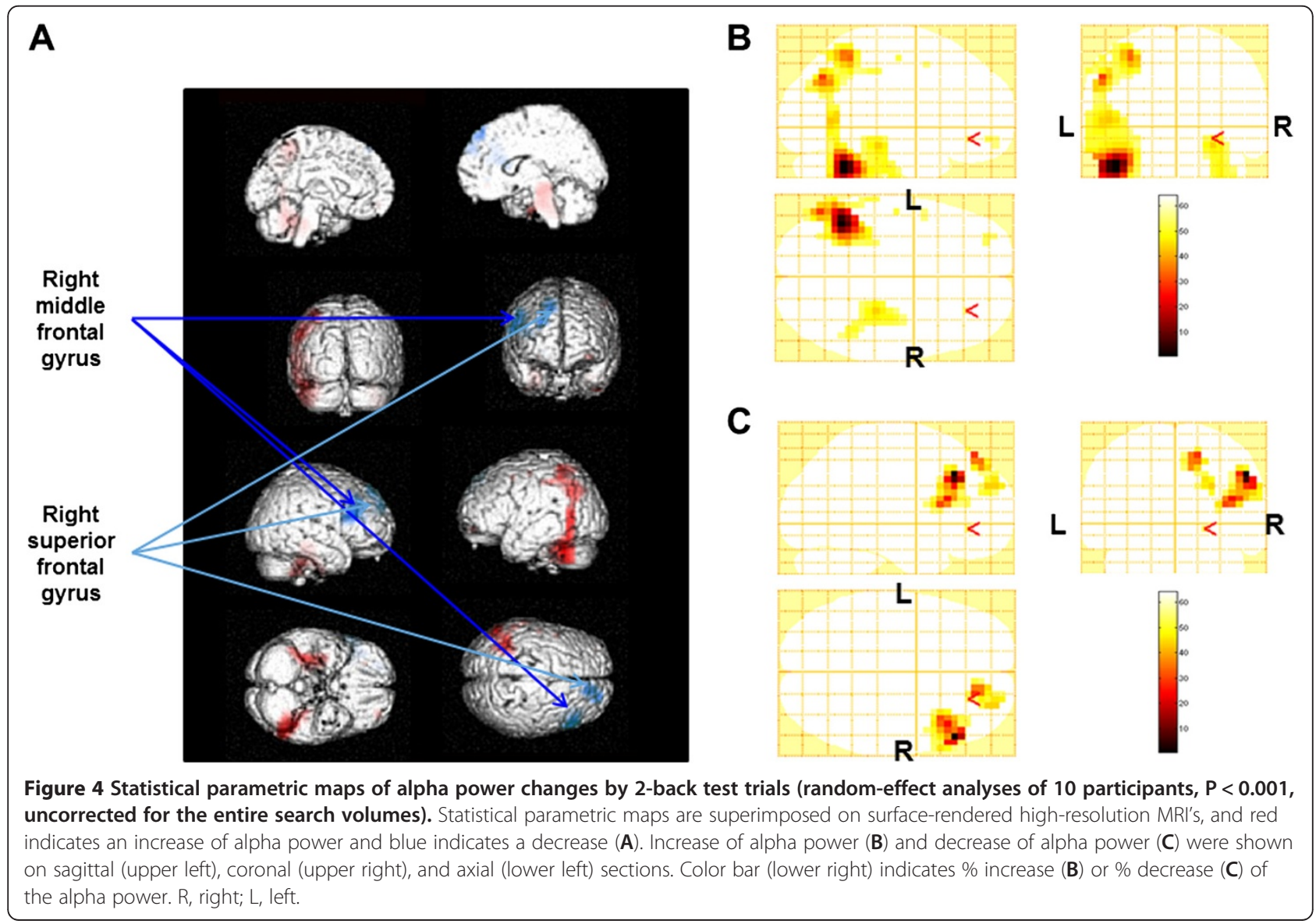




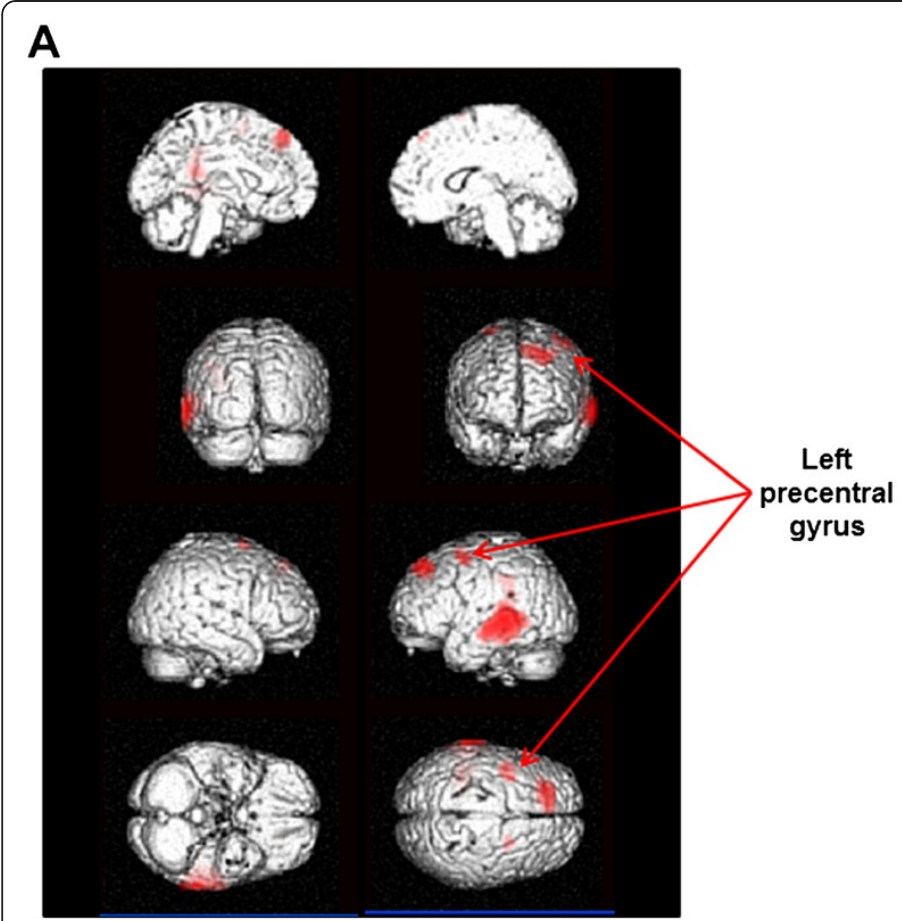

B
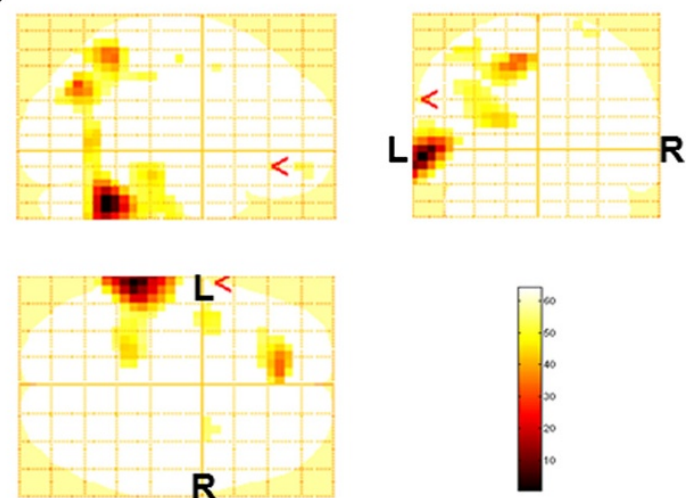

Figure 5 Statistical parametric maps of beta power changes by 0 -back test trials (random-effect analyses of 10 participants, P < 0.001 , uncorrected for the entire search volumes). Statistical parametric maps are superimposed on surface-rendered high-resolution MRI's, and red indicates an increase of alpha power (A). Increase of alpha power (B) was shown on sagittal (upper left), coronal (upper right), and axial (lower left) sections. Color bar (lower right) indicates \% increase of the beta power. R, right; L, left.

temporal, postcentral, and frontal gyrus. In contrast, after the 2-back test, decreased alpha power was shown in the frontal gyrus and increased levels were shown in the parietal lobule, parahippocampal gyrus, uncus, postcentral gyrus, and frontal gyrus. For beta power, after the 0-back test, increased power was shown in the temporal, frontal, cingulate, and precentral gyrus. After the 2-back test, decreased power was shown in the frontal gyrus and increased power was shown in the temporal gyrus and parietal lobule. In addition, the beta power change in the left precentral gyrus was negatively associated with task performance of the 0-back test session; and the alpha power changes in the right middle and superior frontal gyrus were negatively associated with the task performance of 2-back test session.

In our previous EEG study, beta power densities on the P3, Pz, and O1 electrodes and alpha power densities on the $\mathrm{P} 3, \mathrm{Pz}, \mathrm{O} 1$, and $\mathrm{O} 2$ electrodes were decreased after a fatigue-inducing 2-back test session, while alpha power densities on the $\mathrm{P} 3$ and $\mathrm{Pz}$ electrodes were decreased after a fatigue-inducing 0-back test session. Although there are some similarities between the results of the EEG and MEG studies, the differences are apparent. EEG measures electrical fields, which are based on the difference in potentials between an EEG electrode and a reference electrode. In addition, the EEG signal is influenced by the electrical conductivity of intervening tissues such as the skull. In contrast, MEG measures magnetic fields, which does not use a reference, and MEG provides superior spatial resolution and signal-tonoise ratio relative to EEG. Therefore, MEG could identify accurate changes of the mental fatigue-related oscillatory brain activities that were not evident in our previous EEG study.

Multiple, broadly distributed, and continuously interacting dynamic neural networks are achievable through the synchronization of oscillations at particular time-frequency bands. Alpha, one of the large-scale rhythmic oscillations in the brain, is generated in the process of interactions between thalamocortical neurons and GABAergic $(\gamma$-aminobutyric acid) cells in the thalamic reticular nucleus $[25,26]$. This time-frequency band is related to complex cognitive processes such as attention, memory, and mental imagery [27-29]. Chaudhuri and Behan proposed that the fatigue is related to the activation of the thalamo-frontal feedback loops $[5,28,30]$, and the overactivation of the thalamofrontal feedback loops in order to compensate for the functional loss caused by fatigue has been reported in fatigue with some diseases such as multiple sclerosis [31-33] and chronic fatigue syndrome [34,35]. Since mental fatigue induced by 2-back trials suppressed spontaneous alpha power, i.e., desynchronization due to intrinsic events, in 


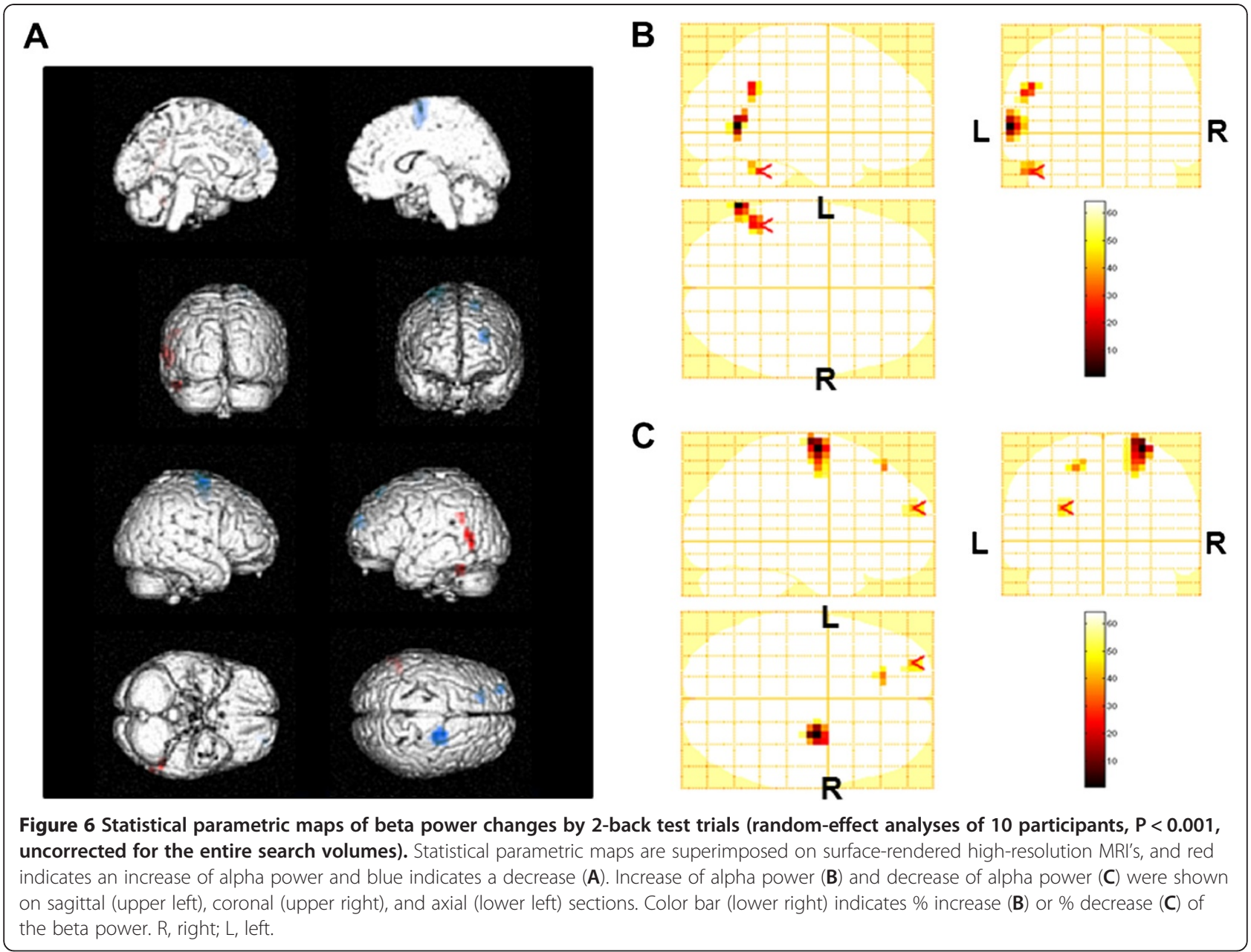

the frontal gyrus, this desyncronization might have some relationships with the activation of the thalamo-frontal feedback loops.

The motor cortex exhibits resting-state synchronization of the oscillations at beta frequency band [36,37]. It has been demonstrated that these oscillations appear to be under the direct control of GABAergic modulation $[38,39]$. These oscillations are facilitated by increasing the inhibitory drive of GABAergic interneurons via GABA-A receptors, which lengthens the inhibitory post-synaptic potential decay time, thereby reducing the frequency of the locally oscillating neuronal network population.
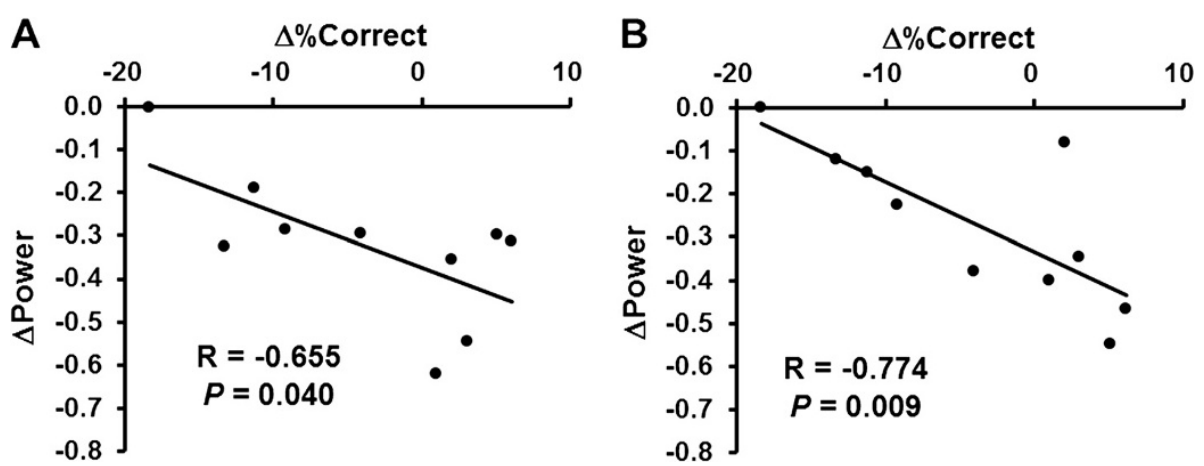

Figure 7 Relationships between change of rate of correct performance [ $\Delta \%$ Correct] during the time course of the 2-back test trials and alpha power change ( $\Delta$ Power) in the right middle frontal gyrus (Brodmann's area 46 ) $(A)$ and in the right superior frontal gyrus (Brodmann's area 9) (B). Linear regression lines, Pearson's correlation coefficients, and $P$ values are shown. 

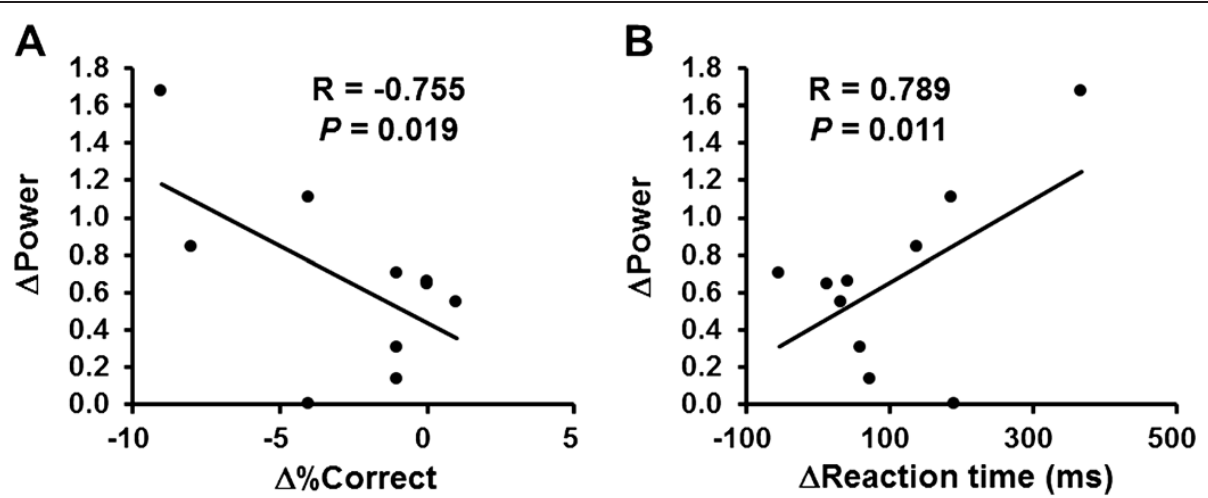

Figure 8 Relationships between changes of rate of correct performance $[\Delta \%$ Correct] $(A)$ and reaction time ( $\Delta$ Reaction time) (B) during the time course of the 0-back test session and beta power change ( $\Delta$ Power) in the left precentral gyrus (Brodmann's areas 4,6$)$. Linear regression lines, Pearson's correlation coefficients, and $\mathrm{P}$ values are shown.

Consequently, this serves to facilitate the recruitment of principal cells to the oscillating population, giving rise to an increase in the amplitude of the oscillatory power, as the participating neuronal pool is increased [40]. In contrast with the results of the 2-back test, the beta power in the left precentral gyrus was increased after 0-back test trials. Interestingly, the increased power in the left precentral gyrus was negatively associated with the percentage correct and positively associated with the reaction time of the 0-back test session. Inhibitory mechanisms apparently suppressed the beta power in the motor areas according to the level of impaired task performance, i.e., mental fatigue. Fatigue is a bio-alarm that senses risks, warns the organism, and orders rest. Therefore, some inhibitory mechanisms that order rest to in order to avoid overwork and even homeostatic catastrophe may exist, and we may recognize that the inhibition is caused by fatigue, resulting in a sensation of fatigue [41]. The increase in inhibitory input to the motor cortex with physical fatigue has been suggested in behavioral [42] and MEG [43] studies; enhanced and persistent activation of the inhibitory system may be related to the pathophysiology of chronic fatigue $[44,45]$. Since this inhibitory system alone causes impaired task performance or even cancellation of task trials, some compensatory mechanisms would be necessary to maintain the task performance. The 0-back test did not require a higher level of mental load; thus, compensatory mechanisms may not be necessary to maintain task performance during the task trials, overactivation of the inhibitory system seems to play a central role in the pathophysiology of mental fatigue caused by 0 -back test trials, in contrast to the mental fatigue caused by 2-back test trials.

\section{Limitations}

While the results of the present study are suggestive of the mechanisms of mental fatigue, only a limited number of participants and limited time-frequency bands were tested. To generalize our results, studies involving a larger number of participants and a variety of time-frequency bands will be needed. In addition, we did not measure the objective markers of mental fatigue in urine and serum [46] and did not investigate the relationship between the MEG data and these markers. Furthermore, although the participants did not complain of physical fatigue, we could not exclude the possibility that the MEG data was affected by physical fatigue. Finally, the use of the task with more burdens, such as 3-back or more, longer duration of tasks could effectively extract the fatigue as the difference of these two conditions. We used 30-min 2-back test as a fatigue-inducing task based on the results of our previous study [7]: To evaluate mental fatigue, participants completed advanced trailmaking test (ATMT [11]) for $30 \mathrm{~min}$, and after the 2back test session for $30 \mathrm{~min}$. ATMT performance was impaired, and this session was shown to be mental fatigue-inducing tasks. In the ATMT, circles numbered from 1 to 25 were randomly located on the display of a personal computer, and the participants were required to use a computer mouse to click the center of the circles in sequence, starting with circle number 1 . The task performance was evaluated by number of errors. In addition, by using the 0-back and 2-back tasks, we could extract the fatigue as the difference related to the MEG responses to visual stimuli of these two conditions $[47,48]$. Therefore, we adopted 30-min 2-back test as a fatigue-inducing task.

\section{Conclusions}

We identified mental fatigue-related changes in MEG spontaneous oscillatory activities. In particular, the beta power change in the left precentral gyrus (BA 4,6) was negatively associated with the task performance for the 0 -back test, and the alpha power changes in the right 
middle (BA 46) and superior (BA 9) frontal gyrus were negatively associated with task performance for the 2-back test. Two types of mental fatigue produced different types of the alterations of the oscillatory brain activities. We believe that our results contribute to providing new perspectives on the neural mechanisms underlying mental fatigue as well as developing evaluation methods and establishing a basis for treatment in order to overcome fatigue.

\section{Abbreviations}

ANOVA: Analysis of variance; ATMT: Advanced trail making test; BA: Brodmann; BRAM: Brain Rhythmic Analysis for Magnetoencephalography; EEG: Electroencephalography; GABA: $\gamma$-aminobutyric acid; MEG: Magnetoencephalography; MNI: Montreal Neurological Institute; MRI: Magnetic resonance imaging; SPM: Statistical parametric mapping; VAS: Visual analogue scale.

\section{Competing interests}

The authors declare that they have no competing interests.

\section{Authors' contributions}

MT took part in planning and designing the experiment, collected the data, performed the data analyses and drafted the manuscript. YS, Al, EK, and MF took part in planning and designing the experiment, collected the data, and performed the data analyses. YW took part in the planning and designing the experiment and helped drafting the manuscript. All authors read and approved the final manuscript.

\section{Acknowledgments}

We thank Manryoukai Imaging Clinic for MRI scans. We thank Forte Science Communication for editorial assistance with the manuscript. This work was supported in part by the Grant-in-Aid for Scientific Research B (KAKENHI: 23300241) from Ministry of Education, Culture, Sports, Science and Technology (MEXT) of Japan and by the grant from Ministry of Health, Labor and Welfare of Japan. The funders had no roles in study design, data collection and analysis, decision to publish, or preparation of the manuscript.

\section{Author details}

'Department of Physiology, Osaka City University Graduate School of Medicine, 1-4-3 Asahimachi, Abeno-ku, Osaka City, Osaka 545-8585, Japan. ${ }^{2}$ Digital \& Network Technology Development Center, Panasonic Co., Ltd, 1006 Kadoma, Kadoma City, Osaka 571-8501, Japan. ${ }^{3}$ RIKEN Center for Molecular Imaging Science, 6-7-3 Minatojima-minamimachi, Chuo-kuKobe City, Hyogo 650-0047, Japan.

Received: 3 October 2012 Accepted: 23 December 2012 Published: 10 January 2013

\section{References}

1. Boksem MA, Tops M: Mental fatigue: costs and benefits. Brain Res Rev 2008, 59(1):125-139.

2. Watanabe $Y$ : Preface and mini-review: fatigue science for human health. In Fatigue science for human health. Edited by Watanabe Y, Evengård B, Natelson BH, Jason LA, Kuratsune H. New York: Springer; 2008:5-11.

3. Tajima S, Yamamoto S, Tanaka M, Kataoka Y, Iwase M, Yoshikawa E, Okada $H$, Onoe $H$, Tsukada $H$, Kuratsune H, Ouchi $Y$, Watanabe Y: Medial orbitofrontal cortex is associated with fatigue sensation. Neurol Res Int 2010, 201:671421.

4. Grandjean E: Fatigue in industry. Br J Ind Med 1979, 36(3):175-186.

5. Chaudhuri A, Behan PO: Fatigue in neurological disorders. Lancet 2004, 363(9413):978-988.

6. Williamson A, Lombardi DA, Folkard S, Stutts J, Courtney TK, Connor JL: The link between fatique and safety. Accid Anal Prev 2011, 43(2):498-515.

7. Mizuno K, Watanabe Y: Utility of an advanced trail making test as a neuropsychological tool for an objective evaluation of work efficiency during mental fatigue. In Fatigue science for human health. Edited by Watanabe Y, Evengård B, Natelson BH, Jason LA, Kuratsune H. New York: Springer; 2008:47-54
8. Tanaka M, Ishii A, Shigihara Y, Tajima S, Funakura M, Kanai E, Watanabe Y: Impaired selective attention caused by mental fatigue. J Neurol Sci (Turkish), in press.

9. Shigihara Y, Tanaka M, Ishii A, Tajima S, Kanai E, Funakura M, Watanabe Y: Two different types of mental fatigue produce different styles of task performance. Neurol Psychiatr Brain Res, in press.

10. Braver TS, Cohen JD, Nystrom LE, Jonides J, Smith EE, Noll DC: A parametric study of prefrontal cortex involvement in human working memory. Neuroimage 1997, 5(1):49-62.

11. Kajimoto O: Development of a method of evaluation of fatigue and its economic impacts. In Fatigue Science for Human Health. Edited by Watanabe Y, Evengård B, Natelson BH, Jason LA, Kuratsune H. New York: Springer; 2008:33-46.

12. Lal SKL, Craig A: A critical review of the psychophysiology of driver fatigue. Biol Physiol 2001, 55(3):173-194.

13. von Stein A, Sarnthein J: Different frequencies for different scales of cortical integration: from local gamma to long range alpha/theta synchronization. Int J Psychophysiol 2000, 38(3):301-313.

14. Tanaka M, Shigihara Y, Ishii A, Funakura M, Kanai E, Watanabe Y: Effect of mental fatigue on the central nervous system: an electroencephalography study. Behav Brain Funct, in press.

15. Stam CJ: Use of magnetoencephalography (MEG) to study functional brain networks in neurodegenerative disorders. J Neurol Sci 2010, 289(1-2):128-34.

16. Oldfield RC: The assessment and analysis of handedness: the Edinburgh inventory. Neuropsychologia 1971, 9(1):97-113.

17. Lee KA, Hicks G, Nino-Murcia G: Validity and reliability of a scale to assess fatigue. Psychiatry Res 1991, 36(3):291-298.

18. Dalal SS, Guggisberg AG, Edwards E, Sekihara K, Findlay AM, Canolty RT, Berger MS, Knight RT, Barbaro NM, Kirsch HE, Nagarajan SS: Fivedimensional neuroimaging: Localization of the time-frequency dynamics of cortical activity. Neurolmage 2008, 40(4):1686-1700.

19. Evans AC, Kamber M, Collins DL, MacDonald D: An MRI-based probablistic atlas of neuroanatomy. In Magnetc resonance scanning and epilepsy. Edited by Shorvon SD. New York: Plenum Press; 1994:263-274.

20. Friston KJ, Holmes AP, Worsley KJ: How many subjects constitute a study? Neurolmage 1999, 10(1):1-5.

21. Lancaster JL, Woldorff MG, Parsons LM, Liotti M, Freitas CS, Rainey L, Kochunov PV, Nickerson D, Mikiten SA, Fox PT: Automated Talairach atlas labels for functional brain mapping. Hum Brain Mapp 2000, 10(3):120-131.

22. Brookes MJ, Wood JR, Stevenson CM, Zumer JM, White TP, Liddle PF, Morris PG: Changes in brain network activity during working memory tasks: a magnetoencephalography study. Neuroimage 2011, 55(4):1804-1815.

23. Varela F, Lachaux JP, Rodriguez E, Martinerie J: The brainweb: phase synchronization and large-scale integration. Nat Rev Neurosci 2001, 2(4):229-239.

24. Schnitzler A, Gross J: Normal and pathological oscillatory communication in the brain. Nat Rev Neurosci 2005, 6(4):285-296.

25. Lopes DA, Silva F: Neural mechanisms underlying brain waves: from neural membranes to networks. Electroencephalogr Clin Neurophysiol 1991, 79(2):81-93

26. Rinzel J, Terman D, Wang $X$, Ermentrout B: Propagating activity patterns in large-scale inhibitory neuronal networks. Science 1998, 279(5355):1351-1355.

27. Gevins AS, Schaffer RE: A critical review of electroencephalographic (EEG) correlates of higher cortical functions. Crit Rev Bioeng 1980, 4(2):113-164.

28. Tesche CD, Uusitalo MA, IImoniemi RJ, Kajola MJ: Characterizing the local oscillatory content of spontaneous cortical activity during mental imagery. Brain Res Cogn Brain Res 1995, 2(4):243-249.

29. Klimesch W: EEG alpha and theta oscillations reflect cognitive and memory performance: a review and analysis. Brain Res Brain Res Rev 1999, 29(2-3):169-195.

30. Chaudhuri A, Behan PO: Fatigue and basal ganglia. J Neurol Sci 2000, 179 (1-2):34-42.

31. Filippi M, Rocca A, Colombo B, Falini A, Codella M, Scotti G, Comi G: Functional magnetic resonance imaging correlates of fatigue in multiple sclerosis. Neuroimage 2002, 15(3):559-567.

32. DeLuca J, Genova HM, Hillary FG, Wylie G: Neural correlates of cognitive fatigue in multiple sclerosis using functional MRI. J Neurol Sci 2008, 270 (1-2):28-39.

33. White AT, Lee JN, Light AR, Light KC: Brain activation in multiple sclerosis: a BOLD fMRI study of the effects of fatiguing hand exercise. Mult Scler 2009, 15(5):580-586. 
34. de Lange FP, Kalkman JS, Bleijenberg G, Hagoort P, van der Werf SP, van der Meer JW, Toni I: Neural correlates of the chronic fatigue syndromean fMRI study. Brain 2004, 127(9):1948-1957.

35. Lange G, Steffener J, Cook DB, Bly BM, Christodoulou C, Liu WC, Deluca J, Natelson $\mathrm{BH}$ : Objective evidence of cognitive complaints in Chronic Fatigue Syndrome: a BOLD fMRI study of verbal working memory. Neuroimage 2005, 26(2):513-524.

36. Murthy VN, Fetz EE: Oscillatory activity in sensorimotor cortex of awake monkeys: synchronization of local field potentials and relation to behavior. J Neurophysiol 1996, 76(6):3949-3967.

37. Baker SN, Olivier E, Lemon RN: Coherent oscillations in monkey motor cortex and hand muscle EMG show task-dependent modulation. J Physiol 1997, 501(1):225-241.

38. Jensen O, Goel P, Kopell N, Pohja M, Hari R, Ermentrout B: On the human sensorimotor-cortex beta rhythm: sources and modeling. Neuroimage 2005, 26(2):347-355.

39. Yamawaki N, Stanford IM, Hall SD, Woodhall GL: Pharmacologically induced and stimulus evoked rhythmic neuronal oscillatory activity in the primary motor cortex in vitro. Neuroscience 2008, 151(2):386-395.

40. Hall SD, Stanford IM, Yamawaki N, McAllister CJ, Rönnqvist KC, Woodhall GL, Furlong PL: The role of GABAergic modulation in motor function related neuronal network activity. Neuroimage 2011, 56(3):1506-1510.

41. Tanaka $M$, Watanabe $Y:$ A new hypothesis of chronic fatigue syndrome: co-conditioning theory. Med Hypotheses 2010, 75(2):244-249.

42. Tsutsumi K, Tanaka M, Shigihara Y, Watanab Y: Central regulation of physical fatigue via mirror visual feedback. Eur J Sport Sci 2011, 11(3):171-175

43. Tanaka M, Shigihara $Y$, Watanabe $Y$ : Central inhibition regulates motor output during physical fatigue. Brain Res 2011, 1412:37-43.

44. Tanaka M, Sadato N, Okada T, Mizuno K, Sasabe T, Tanabe HC, Saito DN, Onoe $H$, Kuratsune $H$, Watanabe $Y$ : Reduced responsiveness is an essential feature of chronic fatigue syndrome: a fMRI study. BMC Neurol 2006, 22(6):9.

45. Tanaka M, Watanabe $Y$ : Supraspinal regulation of physical fatigue. Neurosci Biobehav Rev 2012, 36(1):727-773.

46. Nozaki S, Tanaka M, Mizuno K, Ataka S, Mizuma H, Tahara T, Sugino T, Shirai T, Eguchi A, Okuyama K, Yoshida K, Kajimoto Y, Kuratsune H, Kajimoto O, Watanabe $Y$ : Mental and physical fatigue-related biochemical alterations. Nutrition 2009, 25(1):51-57.

47. Shigihara Y, Tanaka M, Mizuno K, Ishii A, Yamano E, Funakura M, Kanai E, Watanabe $Y$ : Effects of daily levels of fatigue and acutely induced fatigue on the visual evoked magnetic response. Brain Res 2012, 1457:44-50.

48. Tanaka M, Shigihara Y, Funakura M, Kanai E, Watanabe Y: Fatigueassociated alterations of cognitive function and electroencephalographic power densities. PLoS One 2012, 7(4):34774.

doi:10.1186/1744-9081-9-2

Cite this article as: Shigihara et al:: Two types of mental fatigue affect spontaneous oscillatory brain activities in different ways. Behavioral and Brain Functions 2013 9:2.

\section{Submit your next manuscript to BioMed Central and take full advantage of:}

- Convenient online submission

- Thorough peer review

- No space constraints or color figure charges

- Immediate publication on acceptance

- Inclusion in PubMed, CAS, Scopus and Google Scholar

- Research which is freely available for redistribution 